\title{
Cognitive Radio Spectrum Assignment Based on Invasive Weed Optimization Algorithm
}

\author{
Wu Xie ${ }^{1, *}$, Xiao $\mathrm{Li}^{1,2}$, Chuanji Zhu ${ }^{1}$ and Liangjie Yang ${ }^{1}$ \\ ${ }^{1}$ Information and Communication Engineering College, Harbin Engineering University, Harbin, China \\ ${ }^{2}$ Beijing Asimco Tianwei Oil Pump Oil Nozzle Co. LTD, Beijing, China \\ ${ }^{*}$ Corresponding author
}

\begin{abstract}
According to the problem of the optimization of overall performance of idle spectrum assignment for cognitive radio, it proposed a spectrum assignment method for cognitive radio based on binary weed algorithm. Considering maximizing total network efficiency and fair effectiveness as a criterion, the algorithm is designed and implementation steps are presented. Simulation and analysis compares the solution of the spectrum assignment with Genetic Algorithm and particle swarm algorithm. The results show that the spectrum assignment algorithm based on binary invasive weed algorithm has a higher performance. It can achieve maximizing sum reward and proportional fair better and receiving a better optimal solution.
\end{abstract}

Keywords-cognitive radio; spectrum assignment; invasive weed optimization algorithm; graph coloring

\section{INTRODUCTION}

The developing of wireless communication technology has leaded a growing demand for spectrum resources. While the spectrum resources are very limited, however, a large portion of the assigned spectrum is used by authorized user, which has cause large of spectrum be wasted and less spectrum utilization at the same time. The limited available spectrum and the inefficiency in the spectrum usage necessitate a new communication paradigm to exploit the existing wireless spectrum opportunistically. Cognitive radio (CR) technology was proposed to solve those problems [1-3]. Spectrum assignment is a key technology in CR. After sensing the idle spectrum, it can decide whether cognitive radio users (nonauthorized) can utilize those idle channels to communicate or not. If no interface to the authorized users, idle channel could be occupied by cognitive radio users.

Existing spectrum allocation methods are game theorybased spectrum assignment model[4-6], graph coloring theorybased spectrum assignment model[7-9] and auction theorybased spectrum assignment model[10-12] and etc.. Although the model based on game theory and auction theory is very complete, but difficult to execute, while due to the fast convergence speed, execute easily and flexible expanding, the model based on graph coloring theory has caused wide research. Then, a list coloring theory spectrum assignment algorithm was proposed by [13], and then color sensitive graph coloring algorithm was proposed by [8], which has proved that spectrum assignment satisfied the constraint conditions of NP problem, and analyzed its sum reward and proportional fair.
Taking full advantages of Invasive weed optimization algorithm (IWO), based on the model of graph theory in spectrum assignment, a new cognitive radio spectrum assignment was proposed. Though the comparison and simulation, it is verified superiority.

\section{COGNITIVE RADIO SPECTRUM ASSIGNMENT MODEL}

Cognitive radio spectrum assignment model can be described by Channel availability matrix, Channel reward matrix, Interference constraint matrix and Conflict free channel assignment matrix. Assuming there are the $N$ cognitive users in the network, $M$ is the number of available bands which cognitive user can be used.

\section{A. Channel Availability: L}

$$
\boldsymbol{L}=\left\{\boldsymbol{l}_{\boldsymbol{n}, \boldsymbol{m}} \mid \boldsymbol{l}_{\boldsymbol{n}, \boldsymbol{m}} \in\{0,1\}\right\}_{N \times M}
$$

$\boldsymbol{L}$ is using to describe current spectrum whether can be taken use. $\boldsymbol{l}_{n, m}=1$ denotes that channel $m$ is available for cognitive users $n$. In the same way, $\boldsymbol{l}_{n, m}=0$ denotes that cognitive users $n$ cannot occupy spectrum $m$.

\section{B. Channel Reward: $\boldsymbol{B}$}

Because of the different environment cognitive users, and different transmission power and modem techniques cognitive users taking use, different Channel reward cognitive users may get. Matrix $\boldsymbol{B}=\left\{\boldsymbol{b}_{n, m}\right\}_{N{ }_{1} M}$ is utilized to denote Channel reward of cognitive users by occupying current spectrum. $\boldsymbol{b}_{n, m}$ denotes the maximum bandwidth or throughput that can be acquired when user $n$ using spectrum $m$.

\section{Interference Constraint: $\boldsymbol{C}$}

For a certain available bandwidth, if different cognitive users use the frequency band at the same time, they may interfere with each other.

$$
\boldsymbol{C}=\left\{\boldsymbol{c}_{n, k, m} \mid \boldsymbol{c}_{n, k, m} \in\{0,1\}\right\}_{N \times N \times M},
$$

Matrix $\boldsymbol{C}$ denotes interference constraint. Where $\boldsymbol{C}_{n, k, m}=1$ denotes when users $n$ and $k$ using idle spectrum $m$ at the same time would produce interference. Meanwhile $\boldsymbol{C}_{n, k, m}=0$ denotes that users $m$ and $k$ at the same time using spectrum $m$ would not produce interference. When $n$ and $k$ are equivalent $\boldsymbol{C}_{n, k, m}=1$ $\boldsymbol{l}_{n, m}$. 


\section{Conflict-free Channel Assignment: $\boldsymbol{A}$}

The purpose of spectrum assignment is to allocate idle spectrum to cognitive users without interference. $\boldsymbol{A}$ is conflictfree channel assignment $\boldsymbol{A}=\left\{\boldsymbol{a}_{n, m} \mid \boldsymbol{a}_{n, m} \in\{0,1\}\right\}_{N \times M}$, where $\boldsymbol{a}_{n, m}=1$. Meanwhile, $\boldsymbol{a}_{n, m}=0$ denotes channel $m$ cannot be assigned to user $n$ without interference. The conflict free assignment needs to satisfy all the interference constraints defined by $\boldsymbol{C}$, that is

$$
a_{n, m}+a_{k, m} \leq 1, c_{n, k, m}=1, \forall 0 \leq n, k<N, 0 \leq \boldsymbol{m}<\boldsymbol{M}
$$

\section{BINARY INVASIVE WEED OPTIMIZATION ALGORITHM}

\section{A. Invasive Weed Optimization Algorithm}

Invasive weed optimization algorithm is proposed by Mehrabian and Luca in 2006 [14][15]. It is a population-based numerical optimization method and can effectively solve the complicated nonlinear engineering problems. IWO's processes are: Initialize a population, Reproduction and Spatial dispersal.

In the Initialization part, the numbers of the generated seeds is determined by (2).

$$
N_{\text {seed }}=\frac{\left(S_{\max }-S_{\text {min }}\right)}{\left(f_{\max }-f_{\min }\right)}\left(f-f_{\max }\right)+S_{\text {max }}
$$

It can be seen that the number of seeds each plant produce increases linearly from minimum possible seed production to its maximum. In other words, a plant will produce seeds based on its fitness, the colony's lowest fitness and highest fitness to make sure the increase is linear. $\boldsymbol{N}_{\text {seed }}$ denotes the number of seeds. $f$ is the current fitness value. Where, $f_{\min }$ is the minimum fitness of the population in each generation. $f_{\max }$ is the maximum fitness of the population in each generation. $S_{\max }$ is the maximum number of seeds. $S_{\min }$ is the minimum number of seeds.

Spatial dispersal, the produced seeds are being distributed randomly in the problem space by normal distribution with mean zero and a variance parameter decreasing over time $N\left(0, \sigma_{\mathrm{i}}^{2}\right)$. By setting the mean parameter equal to zero, the seeds are located near to the parent plant, distribution equation is:

$$
\sigma_{i}=\left(i_{\max }-n\right)^{n}\left(\sigma_{i n i}-\sigma_{f i n}\right) / i_{\max }^{n}+\sigma_{f i n}
$$

Where, $\boldsymbol{\sigma}_{i n i}$ and $\boldsymbol{\sigma}_{f i n}$ are the initial value of standard deviation and final value of standard distribution. $n$ is the nonlinear modulation index, $i_{\max }$ is the maximum number of iterations, $i$ is the current iteration, $\boldsymbol{\sigma}_{i}$ is the standard deviation at present time step.

\section{B. Binary Invasive Weed Optimization Algorithm}

The weed population is consists by a group of plants with the same structure. Each plants can be represented by $\boldsymbol{P}_{i}=\left[p_{i 1}, p_{i 2}, \cdots, p_{i S}\right]^{T} . S$ shows optimization problem dimension. It is different form invasive weed optimization algorithm because the value of the $p_{i 1}$ only can be 1 or $0[16]$. It has the same steps compare with the traditional IWO. Its main difference from IWO is that in spatial diffusion. After reproduced by a certain number of seeds, traditional IWO generate new plants though:

$$
\text { Xnew }_{j}=P_{i}+N\left(0, \sigma_{i}^{2}\right)^{D},(1 \leq j \leq S)
$$

Where $\boldsymbol{P}_{i}$ denotes parent plants and $X n e w_{j}$ denotes new plants, while binary invasive weed generate new plants though Sigmoid function.

In spectrum assignment model, each individual is a vector, which denotes a feasible solution of spectrum assignment. In order to reduce the computing time, setting $S$ is the numbers of element one in the matrix $\boldsymbol{L}$, only replacing element one that exist in $\boldsymbol{L}$ by individual vector consisting matrix $\boldsymbol{A}$ to generate a new spectrum assignment. Figure 1 is given to explain how it works, where $\boldsymbol{M}=4, \boldsymbol{N}=6$. It can be seen, the dimension of each plant is $\boldsymbol{M} \times \boldsymbol{N}=24$, while the number of one element is 10 , utilizing this method has cut the search area of optimal solution from $2^{24}$ to $2^{10}$ which has compress search area $2^{12}$ times in this model. When the model has a large $\boldsymbol{M}, \boldsymbol{N}$, the ability of compression space is considerable.

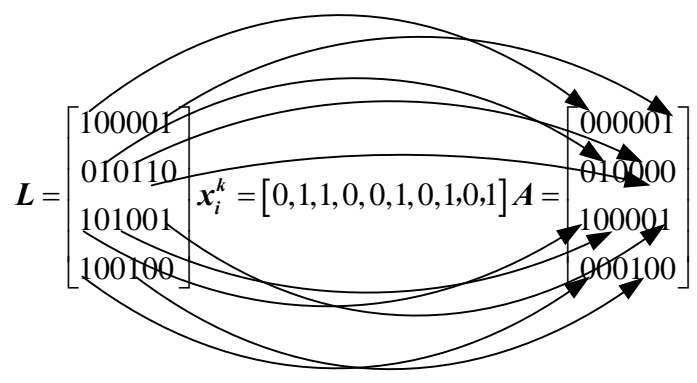

FIGURE I. WEEDS CODING STRUCTURE SAMPLE

According to this model, the weeds of spatial diffusion will be in this way: Firstly, take absolute value of the spatial diffusion value $\boldsymbol{d}_{i}{ }^{\boldsymbol{k}}$. Where $\boldsymbol{k}$ denotes current iteration times and $\boldsymbol{t}$ denotes the $\boldsymbol{t}$ bits in individual $\left(\boldsymbol{P}_{i}\right)$.Then, mapping its value into 0 or 1 though Sigmoid function: $\boldsymbol{f}\left(\boldsymbol{d}_{t}^{k}\right)=\boldsymbol{s i g}\left(\boldsymbol{d}_{t}^{k}-6\right)$.

$$
\hat{\boldsymbol{d}}_{t}^{k}=\left\{\begin{array}{l}
1,\left(\rho<\boldsymbol{f}\left(\boldsymbol{d}_{t}^{k}\right)\right) \\
0, \text { (others) }
\end{array}\right.
$$

Finally, generates new colonies:

$$
p_{t}^{k+1}=\bmod \left(p_{t}^{k}+\hat{d}_{t}^{k}, 2\right)
$$

Fitness function is corresponding to objective function, which can measure the performance of the solution. In this paper objective functions are Max Sum Reward (MSR) and Max Proportional Fair (MPR).

MSR was to make their revenue maximize. The calculating formula is: 


$$
\boldsymbol{F}_{\text {sum }}\left(\boldsymbol{A}^{*}, \boldsymbol{B}\right)=\sum_{n=1}^{N} \sum_{m=1}^{M} \boldsymbol{a}_{n, m}^{*} \cdot \boldsymbol{b}_{n, m}=\sum_{n=1}^{N} \beta_{n}
$$

The average MSR is:

$$
\boldsymbol{F}_{\text {mean }}\left(\boldsymbol{A}^{*}, \boldsymbol{B}\right)=\frac{1}{N} \sum_{n=1}^{N} \sum_{m=1}^{M} \boldsymbol{a}_{n, m}^{*} \cdot \boldsymbol{b}_{n, m}=\frac{1}{N} \sum_{n=1}^{N} \beta_{n}
$$

MPR is used to measure the network of cognitive users to get the fair performance benefits. The calculating formula is:

$$
\boldsymbol{F}\left(\boldsymbol{A}^{*}, \boldsymbol{B}\right)=\left(\prod_{n=1}^{N}\left(\sum_{m=1}^{M} \boldsymbol{a}_{n, m}^{*} \cdot \boldsymbol{b}_{n, m}+10^{-4}\right)\right)^{1 / N}=\left(\prod_{n=1}^{N}\left(\beta_{n}+10^{-4}\right)\right)^{1 / N}
$$

The binary of weeds algorithm procedure in the spectrum assignment model are shown below.

Step1: Initialization: randomly generated initial population $\boldsymbol{p}_{i}, \boldsymbol{P}_{i}=\left[p_{i 1}, p_{i 2}, \cdots, p_{i S}\right]^{T}, i=1,2, \ldots, L$, where $\boldsymbol{p}_{i 1}$ only have two values 0 or 1 . Checking $\boldsymbol{p}_{i}$ whether it meets no interference constraint conditions or not. For each bandwidth $m(0 \leq m<M)$, finding out all $n$ and $k$ which satisfied $\boldsymbol{C}_{n, k, m}=1$, then checking if row $n$ and row $k$ of column $m$ exist more than one 1 . If it does randomly presetting one position to 0 , the other remains the same;

Step2: Calculating the fitness function value of the initial population individuals;

Step3: Reproduction: reproduced by a certain number of seeds through (2);

Step4: Spatial dispersal: The produced seeds in the previous step are being distributed randomly by (5) around the plants, which has best fitness. Before distributed mapping, the diffusion value to binary bits through (4). Then, Checking whether it meets no interference constraint conditions or not;

Step5: After iterations, examining the number of plants, if its maximum eliminate the inappropriate and weaker plants in a competitive manner for limiting the maximum number of plants in a colony, if not go to step 6;

Step6: If iterations reach its maximum, stop the algorithm otherwise go to the step 2 .

\section{SIMULATION RESULTS}

This paper utilizes software Matlab2010b to simulate under the environment of windows. In order to evaluate the capabilities of spectrum assignment by Invasive Weed Optimization algorithm compares with spectrum assignment by genetic algorithm (GA) and spectrum assignment by particle swarm algorithm (PSO). In GA the crossover probability is 0.8 and mutation probability is 0.01 . In PSO the maximum particles speed $\left(\boldsymbol{V}_{\max }\right)$ is 4 and inertia weight is 0.2 , while in IWO set $n$ (nonlinear modulation index) equal 3.

Figure II and Figure III show Sum Reward and Proportional Fair curves between IWO and GA, PSO. Where $M=20, N=22$, with same random matrix $\boldsymbol{L}, \boldsymbol{B}, \boldsymbol{C}$, maximum iteration for each algorithm is 50 . It can be seen that, not only Sum Reward but also Proportional Fair spectrum assignment by IWO has a better capabilities than the other two algorithms.

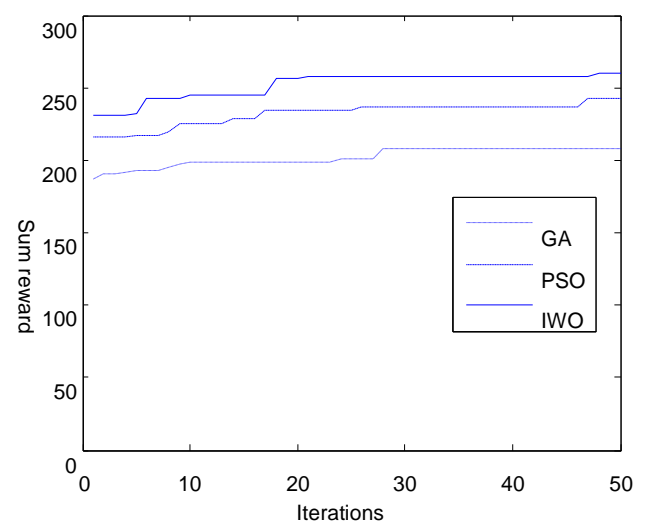

FIGURE II. SUM REWARD WITH SAME RANDOM MATRIX $\boldsymbol{L}, \boldsymbol{B}, \boldsymbol{C}$

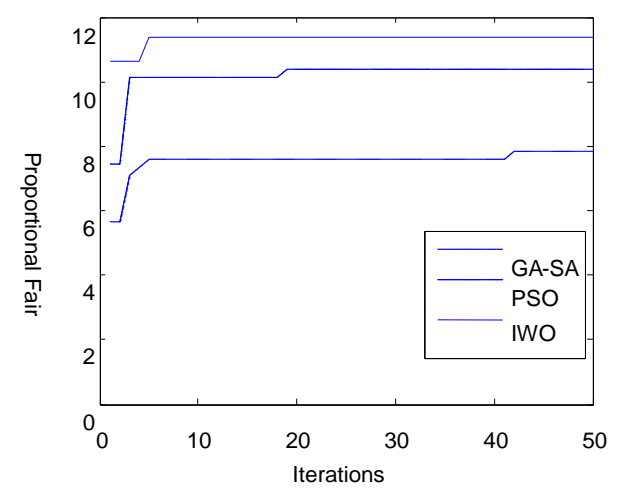

FIGURE III. PROPORTIONAL FAIR CURVES WITH SAME RANDOM MATRIX $\boldsymbol{L}, \boldsymbol{B}, \boldsymbol{C}$

Figure IV and Figure V shows after 50 times of experiment, the capabilities of Sum Reward and Proportional Fair curves of those three algorithms, where the relative parameters $M=20$, $N=22$, with the different matrix $\boldsymbol{L}, \boldsymbol{B}, \boldsymbol{C}$. The results turn out to be that with all the fitness function the IWO shows better performance than the others during the 50 times of experiment.

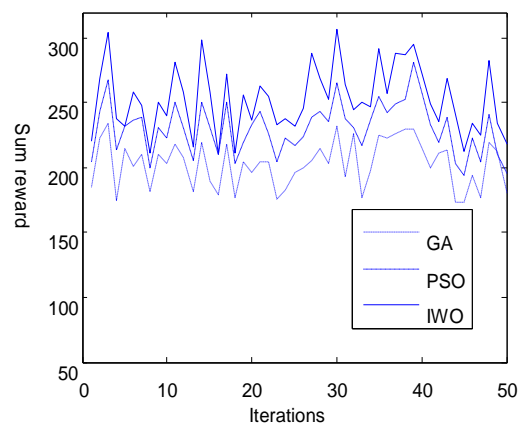

FIGURE IV. SUM REWARD WITH THE DIFFERENT RANDOM MATRIX $\boldsymbol{L}, \boldsymbol{B}, \boldsymbol{C}$ 


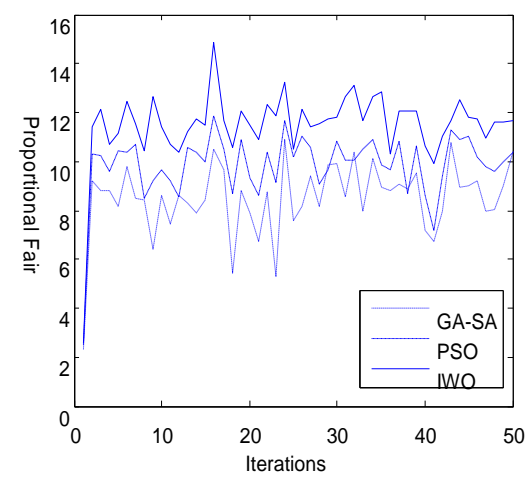

FIGURE V. PROPORTIONAL FAIR CURVES WITH THE DIFFERENT RANDOM MATRIX $\boldsymbol{L}, \boldsymbol{B}, \boldsymbol{C}$

A method of spectrum assignment for cognitive radio based on binary-weed algorithm is proposed. Considering maximizing total network efficiency as a criterion, it improved between cognitive user and user of fairness in spectrum assignment. Simulation and analysis compares the solution of the Spectrum assignment with Genetic algorithm and particle swarm algorithm. The results show that the spectrum assignment algorithm based on binary-weed algorithm has a higher performance. It can achieve maximizing total network efficiency and better fair effectiveness and also conclude a superior solution.

\section{ACKNOWLEDGMENT}

The research of this paper was supported by the Natural Science Foundation of Heilongjiang Province, China (Grant No. F201339).

\section{REFERENCES}

[1] Mitola J, Maguire G Q. Cognitive radio: Making software radios more personal[J]. IEEE Personal Communications, 1999, 6(4): 13-18.

[2] Mitola J. The software radio architecture[J]. IEEE Communications Magazine, 1995, 33(5): 26-38.

[3] Mitola J. Software radio architecture: a mathematical perspective, Selected Areas in Communications [J]. IEEE Journal, 1999, 17(4): 514538.

[4] James Neel, R. Michael Buehrer, Jeffrey H Reed, Robert P. Gilles. Game theoretic analysis of a network of cognitive radios[C]// The 2002 45th Midwest Symposium on Circuits and Systems, 2002.

[5] James Neel, Jeffrey H. Reed, Robert P. Gilles. The Role of Game Theory in the Analysis of Software Radio Networks[C]// SDR Forum Technical Conference, 2002.

[6] James Neel, Jeffrey H. Reed, Robert P. Gilles. Convergence of Cognitive Radio Networks[C]//Wireless Communications and Networking Conference, 2004: 2250-2255.

[7] Peng Chunyi, Zheng Haitao, Zhao B Y. Utilization and fairness in spectrum assignment for opportunistic spectrum access[J] Journal Mobile Networks and Applications, 2006, 11(4): 555-576.

[8] Zheng Haitao, Peng Chunyi. Collaboration and fairness in opportunistic spectrum access[C]// IEEE International Conference on Communications, 2005: 3132-3136.

[9] Wang Fan, Krunz Marwan, Cui Shuguang. Price based spectrum management in cognitive radio networks[J]. IEEE Journal of Selected Topics in Signal Processing, 2007, 2(1): 74-87.
[10] Zhu Ji, Liu K J R. Multi-stage Pricing Game for Collusion-Resistant Dynamic Spectrum Allocation[J]. IEEE Trans. On Communications, 2008, 26(1): 182-191.

[11] Kloeck C, Jaekel H, Jondral F K. Dynamic and Local Combined Pricing, Allocation and Billing System with Cognitive Radios[C]//The First IEEE International Symposium on New Frontiers in Dynamic Spectrum Access Networks. Baltimore, 2005: 73-81.

[12] Yu Yangying, Zhu Jiang, Zhang Shengfeng. Channel Allocation Mechanisms for Cognitive Radio System Based on Repeated Multi-bid Auction [J]. Communications Technology. 2008, 41(5): 75-78.

[13] Wei Wang, Xin Liu. List-Coloring Based Channel Allocation for OpenSpectrum WirelessNetworks[C]//The 62nd IEEE Vehicular Technology Conference, 2005:690-694.

[14] Mehrabian A R, Lucas C. A novel numerical optimization algorithm inspired from weed colonization[J]. Ecological Informatics, 2006, 1(3): 355-366.

[15] Roshanaei M, Lucas C, Mehrabian A R. Adaptive beamforming using a novel numerical optimization algorithm[J]. IET Microwaves, Antennas \& Propagation, 2009, 3(5): 765-773.

[16] Zhang Shuai, Wang Yingguan, Xia Lingnan. Invasive weed optimization algorithm of discrete binary version[J]. Huazhong Univ. of Sci. \& Tech.(Natural Science Edition). 2011, 39(10): 55-60. 